\title{
ON THE DISCRETE-TIME ROBUST NONLINEAR SERVOMECHANISM PROBLEM*
}

\author{
WEIYAO LAN ${ }^{\dagger}$ AND JIE HUANG ${ }^{\dagger}$
}

\begin{abstract}
The paper addresses the robust servomechanism problem for nonlinear discrete-time systems. The solvability conditions for the discrete-time $k^{t h}$-order robust servomechanism problem is first established by using the internal model principle. Then it is further shown that, under an additional assumption on the solution of the discrete regulator equations, the solvability of the discrete-time $k^{t h}$-order robust servomechanism problem leads to the solvability of the discrete-time robust servomechanism problem. The results of this paper give a discrete-time counterpart of the results on the continuous-time robust servomechanism problem.
\end{abstract}

Keywords: discrete-time system, robust control, servomechanism problem, internal model principle.

1. Introduction. Servomechanism problem, alternatively, output regulation problem, is one of the most fundamental problems in control theory. It aims to design a feedback control law for a system such that the output of the system can asymptotically track a class of reference inputs and/or reject a class of disturbances while maintaining the closed-loop stability. Here both the class of reference inputs and the class of disturbances are generated by an autonomous system called exosystem. For linear systems, the problem has been thoroughly studied since 1970s [5], [7] and [8]. For nonlinear systems without uncertainty, the problem is extensively studied since 1990s for both the continuous-time systems [15], [16], [17] and the discrete-time systems [4], [11], [18]. However, the attentions for the robust servomechanism problem, which takes into account the uncertainties in the plant, are only limited to the continuous-time systems [1], [10], [12], [13], [19] and [20]. In particular, the notion of $k^{t h}$-order robust control was introduced in [12] and [13]. The resultant controller is such that, regardless of small parameter perturbations in the plant, the closed-loop system will induce a stable center manifold with the error-map zero up to $k^{t h}$-order at each point of the manifold. The controller features the internal model principle in that it contains an internal model of a system generated by the exosystem. This result coupled with the performance analysis of a $k^{t h}$-order approximate controller as introduced in [15] guarantees that the steady state tracking error of the closed-loop system is of $k^{\text {th }}$-order in the exogenous signal regardless of small parameter perturbations in the plant. Moreover, under an additional assumption on the solution of

${ }^{*}$ Received on July 25, 2003; accepted for publication on October 12, 2003. The work described in this paper was partially supported by a grant from the Research Grants Council of the Hong Kong Special Administration Region (Project No. CUHK4181/01E).

$\dagger$ Department of Automation and Computer-Aided Engineering, The Chinese University of Hong Kong, Hong Kong, E-mail: wylan, jhuang@acae.cuhk.edu.hk 
the regulator equations, the controller that solves the $k^{\text {th }}$-order robust servomechanism problem also solves the robust servomechanism problem. In this paper, we will develop a discrete-time counterpart of the results in [12] and [13] for discrete-time nonlinear systems with parameter uncertainties.

This paper is organized as follows. In Sections 2 , both the discrete-time $k^{t_{-}}$ order robust servomechanism problem and the discrete-time robust servomechanism problem are formulated, and then some of the preliminaries about the linear internal model and the power series solution of the discrete regulator equations are presented. Section 3 establishes the solvability conditions for the discrete-time $k^{t h}$-order robust servomechanism problem. Section 4 shows that, under some additional assumption on the solution of the discrete regulator equations, the solvability of the discrete-time $k^{\text {th }}$-order robust servomechanism problem leads to the solvability of the discrete-time robust servomechanism problem. In Section 5, we illustrate our approach using the inverted pendulum on a cart system, and compare the tracking performance of the closed-loop systems resulting from the $k^{t h}$-order robust controller introduced in this paper and resulting from the $k^{t h}$-order approximate controller introduced in [21]. Finally, Section 6 concludes this paper with some remarks.

2. Problem Formulation and Preliminaries. Consider a discrete-time nonlinear system described by

$$
\begin{aligned}
x(t+1) & =f(x(t), u(t), v(t), w), \quad x(0)=x_{0}, \quad t=0,1,2, \cdots \\
e(t) & =h(x(t), u(t), v(t), w),
\end{aligned}
$$

where $x(t) \in \Re^{n}$ is the plant state, $u(t) \in \Re^{m}$ the plant input, $e(t) \in \Re^{p}$ the plant output representing the tracking error, $w \in \Re^{N}$ the plant uncertain parameters, and $v(t) \in \Re^{q}$ the exogenous signal representing the disturbance and/or the reference input. It is assumed that $v(t)$ is generated by an autonomous system

$$
v(t+1)=a(v(t)), \quad v(0)=v_{0}, \quad t=0,1,2, \cdots .
$$

We will consider the following two classes of feedback control laws, namely,

(i). Dynamic state feedback control law,

$$
\begin{aligned}
u(t) & =\phi(x(t), z(t)) \\
z(t+1) & =g(z(t), e(t)), \quad z(0)=z_{0} .
\end{aligned}
$$

(ii). Dynamic output feedback control law,

$$
\begin{aligned}
u(t) & =\phi(z(t)) \\
z(t+1) & =g(z(t), e(t)), \quad z(0)=z_{0}
\end{aligned}
$$

where $\mathrm{z}(\mathrm{t})$ is the compensator state vector of dimension $n_{z}$ to be specified later. 
Letting $x_{c}=(x, z)$, the closed-loop system under the control law (3) can be written as follows

$$
\begin{aligned}
x_{c}(t+1) & =f_{c}\left(x_{c}(t), v(t), w\right), & & x_{c}(0)=x_{c 0}=\left(x_{0}, z_{0}\right) \\
v(t+1) & =a(v(t)), & & v(0)=v_{0} \\
e(t) & =h_{c}\left(x_{c}(t), v(t), w\right) & &
\end{aligned}
$$

where

$$
\begin{aligned}
f_{c}\left(x_{c}, v, w\right) & =\left[\begin{array}{c}
f(x, \phi(x, z), v, w) \\
g(z, h(x, \phi(x, z), v, w))
\end{array}\right] \\
h_{c}\left(x_{c}, v, w\right) & =h(x, \phi(x, z), v, w) .
\end{aligned}
$$

(5) can also represent the closed-loop system under the dynamic output feedback control law (4) if we replacing $\phi(x, z)$ by $\phi(z)$ in $(6)$.

For simplicity, all the functions involved in this setup are assumed to be sufficiently smooth and defined globally on the appropriate Euclidean spaces, with the value zero at the respective origins. It is also assumed that 0 is the nominal value of the uncertain parameter $w$, and $f(0,0,0, w)=0$ and $h(0,0,0, w)=0$ for all $w \in \Re^{N}$.

The discrete-time $k^{t h}$-order robust servomechanism problem and the discrete-time robust servomechanism problem are formulated as follows.

Discrete-time $k^{t h}$-order Robust Servomechanism Problem. Find a controller of the form (3) or (4) such that the closed-loop system (5) satisfies

R1: The matrix $\frac{\partial f_{c}}{\partial x_{c}}(0,0,0)$ is Schur, i.e., all the eigenvalues of the matrix $\frac{\partial f_{c}}{\partial x_{c}}(0$, $0,0)$ are located strictly inside the unit disk.

R2: For all sufficiently small $x_{c 0}, v_{0}$ and $w$, the trajectory $x_{c}(t)$ of the closed-loop system (5) satisfies

$$
\lim _{t \rightarrow \infty}\left(e(t)-o^{k}(v(t))\right)=0
$$

where $k$ is some given positive integer, and $o^{k}(v)$ is some sufficiently smooth function of $v$ zero up to $k^{\text {th }}$-order .

Discrete-time Robust Servomechanism Problem. Find a controller of the form (3) or (4) such that the closed-loop system (5) satisfies R1 and

R3: For all sufficiently small $x_{c 0}, v_{0}$ and $w$, the trajectory $x_{c}(t)$ of the closed-loop system (5) satisfies

$$
\lim _{t \rightarrow \infty} e(t)=0
$$

In this paper, we make the following assumptions,

A1: The equilibrium of exosystem (2) at the origin is stable at $v=0$ and all the eigenvalues of $\frac{\partial a}{\partial v}(0)$ are located on the unit circle.

A2: The pair $\left\{\frac{\partial f}{\partial x}(0,0,0,0), \frac{\partial f}{\partial u}(0,0,0,0)\right\}$ is stabilizable.

A3: The pair $\left\{\frac{\partial h}{\partial x}(0,0,0,0), \frac{\partial f}{\partial x}(0,0,0,0)\right\}$ is detectable. 
A4: For $l=1,2, \cdots$,

$$
\operatorname{rank}\left[\begin{array}{cc}
\frac{\partial f}{\partial x}(0,0,0,0)-\lambda I & \frac{\partial f}{\partial u}(0,0,0,0) \\
\frac{\partial h}{\partial x}(0,0,0,0) & \frac{\partial h}{\partial u}(0,0,0,0)
\end{array}\right]=n+p
$$

for all $\lambda$ given by

$$
\begin{aligned}
& \left\{\lambda \mid \lambda=\lambda_{1}^{l_{1}} \times \lambda_{2}^{l_{2}} \times \cdots \times \lambda_{q}^{l_{q}}, l_{1}+l_{2}+\cdots+l_{q}=l,\right. \\
& \left.\quad l_{1}, l_{2}, \cdots, l_{q}=0,1,2, \cdots\right\}
\end{aligned}
$$

where $\lambda_{1}, \lambda_{2}, \cdots, \lambda_{q}$ are eigenvalues of the matrix $\frac{\partial a}{\partial v}(0)$.

REMARK 2.1. The two problems defined above are extended, respectively, from the discrete-time $k^{\text {th }}$-order servomechanism problem ([21]) and the discrete-time servomechanism problem ([3], [4], [11] and [14] etc.) by considering the uncertain parameters $w$ in the plant. Assumption A1 is made so that the center manifold theorem for map can be used to guarantee the closed-loop system stability [2]. Assumptions A2 to A3 guarantee that the given plant can be locally stabilized by state feedback and output feedback, respectively. Assumption A4 guarantees that the discrete regulator equations (15) as will be introduced shortly admit a formal power series solution which is crucial for the solvability of the $k^{\text {th }}$-order robust servomechanism problem.

From the results of the discrete-time servomechanism problem in [11], [14] and [21], it is not difficult to deduce that, under assumption A1, and suppose that the close-loop system (5) satisfies R1, then,

(i) the closed-loop system (5) satisfies $R 3$ if there exists a sufficiently smooth function $\mathbf{x}_{c}(v, w)$ with $\mathbf{x}_{c}(0,0)=0$ such that

$$
\begin{aligned}
\mathbf{x}_{c}(a(v), w) & =f_{c}\left(\mathbf{x}_{c}(v, w), v, w\right) \\
0 & =h_{c}\left(\mathbf{x}_{c}(v, w), v, w\right)
\end{aligned}
$$

(ii) the closed-loop system (5) satisfies $R 2$ if there exists a sufficiently smooth function $\mathbf{x}_{c}^{(k)}(v, w)$ with $\mathbf{x}_{c}^{(k)}(0,0)=0$ such that

$$
\begin{aligned}
\mathbf{x}_{c}^{(k)}(a(v), w) & =f_{c}\left(\mathbf{x}_{c}^{(k)}(v, w), v, w\right) \\
o^{k}(v) & =h_{c}\left(\mathbf{x}_{c}^{(k)}(v, w), v, w\right) .
\end{aligned}
$$

Further, Equations (11) and (12) can be made satisfied only if the following assumption hold.

A5: There exist sufficiently smooth functions $\mathbf{x}(v, w)$ and $\mathbf{u}(v, w)$ with $\mathbf{x}(0,0)=0$ and $\mathbf{u}(0,0)=0$ such that for $v \in V, w \in W$,

$$
\begin{aligned}
\mathbf{x}(a(v), w) & =f(\mathbf{x}(v, w), \mathbf{u}(v, w), v, w) \\
0 & =h(\mathbf{x}(v, w), \mathbf{u}(v, w), v, w)
\end{aligned}
$$

where $V \subset \Re^{q}, W \subset \Re^{N}$ are some neighborhoods of the origin of $\Re^{q}$ and $\Re^{N}$, respectively. 
Equations (15) are called the discrete regulator equations.

Next, let us summarize some results about the linear internal model principle described in [5], [8], and [10].

Consider a linear system

$$
\begin{aligned}
x(t+1) & =A x(t)+B u(t)+E v(t) \\
e(t) & =C x(t)+D u(t)+F v(t) \\
v(t+1) & =A_{1} v(t)
\end{aligned}
$$

where $x \in \Re^{n}, u \in \Re^{m}, e \in \Re^{p}$ and $v \in \Re^{q}$, and a pair of constant matrices $\left(\mathcal{G}_{1}, \mathcal{G}_{2}\right)$ of the form

$$
\mathcal{G}_{1}=T\left[\begin{array}{cc}
S_{1} & S_{2} \\
0 & G_{1}
\end{array}\right] T^{-1}, \quad \mathcal{G}_{2}=T\left[\begin{array}{c}
S_{3} \\
G_{2}
\end{array}\right]
$$

where $T$ is a nonsingular matrix with the same dimension of $\mathcal{G}_{1}, S_{1}, S_{2}$, and $S_{3}$ are arbitrary constant matrices with appropriate dimensions, and $G_{1}$ and $G_{2}$ are given by

$$
G_{1}=\text { block } \operatorname{diag}\left\{\beta_{1}, \cdots, \beta_{p}\right\}, \quad G_{2}=\operatorname{block} \operatorname{diag}\left\{\sigma_{1}, \cdots, \sigma_{p}\right\}
$$

where $\beta_{i}$ is constant square matrix of dimension $n_{i}$ and $\sigma_{i}$ is constant column vector of dimension $n_{i}$. For any square matrix $A_{1}$, the pair of matrices $\left(\mathcal{G}_{1}, \mathcal{G}_{2}\right)$ are said to incorporate an internal model of the matrix $A_{1}$ if

(i). $\beta_{i}$ and $\sigma_{i}$ are controllable.

(ii). The minimal polynomial of $A_{1}$ divides the characteristic polynomial of $\beta_{i}$.

(iii). For all $\lambda$ that are the eigenvalues of $G_{1}$,

$$
\operatorname{rank}\left[\begin{array}{cc}
A-\lambda I & B \\
C & D
\end{array}\right]=n+p
$$

REMARK 2.2. If $\left(S_{1}, S_{2}, S_{3}\right)$ are zero dimensional matrices and $T=I$, then the pair $\left(G_{1}, G_{2}\right)$ itself incorporates an internal model of the matrix $A_{1}$.

REMARK 2.3. Given any matrix $A_{1}$, if

$$
\operatorname{rank}\left[\begin{array}{cc}
A-\lambda I & B \\
C & D
\end{array}\right]=n+p
$$

for all $\lambda$ that are the eigenvalues of $A_{1}$, then it is always possible to find an internal model of the matrix $A_{1}$. In fact, let

$$
\alpha_{m}(\lambda)=\lambda^{n_{m}}+\alpha_{1} \lambda^{n_{m}-1}+\cdots+\alpha_{n_{m}-1} \lambda+\alpha_{n_{m}}
$$


be the minimal polynomial of $A_{1}$, and let

$$
\beta_{i}=\left[\begin{array}{cccc}
0 & 1 & \cdots & 0 \\
0 & 0 & \cdots & 0 \\
\vdots & \vdots & \vdots & \vdots \\
0 & 0 & \cdots & 1 \\
-\alpha_{n_{m}} & -\alpha_{n_{m}-1} & \cdots & -\alpha_{1}
\end{array}\right], \quad \sigma_{i}=\left[\begin{array}{c}
0 \\
0 \\
\vdots \\
0 \\
1
\end{array}\right], i=1, \cdots, p
$$

Then, clearly, the pair $\left(G_{1}, G_{2}\right)$ defined by (16) incorporates an internal model of the matrix $A_{1}$.

REMARK 2.4. It is known from [6] that, if $(A, B)$ is stabilizable and $\left(G_{1}, G_{2}\right)$ incorporates an internal model of $A_{1}$, then the pair

$$
\left[\begin{array}{cc}
A & 0 \\
G_{2} C & G_{1}
\end{array}\right], \quad\left[\begin{array}{c}
B \\
G_{2} D
\end{array}\right]
$$

is also stabilizable. As a result, there exist matrices $K_{1}$ and $K_{2}$ such that the matrix $A_{c}$,

$$
A_{c}=\left[\begin{array}{cc}
A+B K_{1} & B K_{2} \\
G_{2}\left(C+D K_{1}\right) & G_{1}+G_{2} D K_{2}
\end{array}\right],
$$

is Schur. Therefore, if $\sigma\left(A_{1}\right) \bigcap \sigma\left(A_{c}\right)=\emptyset$, the following linear matrix equations

$$
\begin{aligned}
X A_{1} & =\left(A+B K_{1}\right) X+B K_{2} Z+E \\
Z A_{1} & =G_{1} Z+G_{2}\left(\left(C+D K_{1}\right) X+D K_{2} Z+F\right)
\end{aligned}
$$

have a unique solution in $X$ and $Z$. Moreover, the solution of (19) is such that [10],

$$
\left(C+D K_{1}\right) X+D K_{2} Z+F=0
$$

Finally, we close this section by giving the solvability conditions of the discrete regulator equations in terms of a power series. For this purpose, the following notation will be defined first.

For any matrix $K$, we will use Kronecker product notation

$$
K^{(0)}=1, \quad K^{(1)}=K, \quad K^{(i)}=\underbrace{K \otimes K \cdots \otimes K}_{\text {i factors }} .
$$

For the $q \times 1$ vector $v$, let $v^{[l]}$ denote the vector

$$
v^{[l]}=\left[\begin{array}{llllllllll}
v_{1}^{l} & v_{1}^{l-1} v_{2} & \cdots & v_{1}^{l-1} v_{q} & v_{1}^{l-2} v_{2}^{2} & v_{1}^{l-2} v_{2} v_{3} & \cdots & v_{1}^{l-2} v_{2} v_{q} & \cdots & v_{q}^{l}
\end{array}\right]^{T} .
$$

It is clear that the dimension of $v^{[l]}$ and $v^{(l)}$ are, respectively,

$$
\left(\begin{array}{c}
q+l-1 \\
l
\end{array}\right) \times 1, \quad q^{l} \times 1
$$


and that there exist matrices of appropriate dimensions such that

$$
\begin{aligned}
v^{[l]} & =M_{l} v^{(l)} \\
v^{(l)} & =N_{l} v^{[l]}
\end{aligned}
$$

where $M_{l} N_{l}$ is an identity matrix.

Then we can expand any smooth functions $f(x, u, v, w), h(x, u, v, w)$ and $a(v)$ in terms of power series as follows,

$$
\begin{aligned}
f(x, u, v, w) & =\sum_{l \geq 1} \sum_{\substack{i+j+k=l \\
i, j, k \geq 0}} F_{i j k}(w) x^{(i)} \otimes u^{(j)} \otimes v^{(k)}, \\
h(x, u, v, w) & =\sum_{l \geq 1} \sum_{\substack{i+j+k=l \\
i, j, k \geq 0}} H_{i j k}(w) x^{(i)} \otimes u^{(j)} \otimes v^{(k)}, \\
a(v) & =\sum_{i \geq 1} A_{i} v^{(i)} .
\end{aligned}
$$

LEMma 2.1. The power series

$$
\begin{aligned}
& \mathbf{x}(v, w)=\sum_{l \geq 1} X_{l w} v^{[l]} \\
& \mathbf{u}(v, w)=\sum_{l \geq 1} U_{l w} v^{[l]}
\end{aligned}
$$

formally satisfies the discrete regulator equations

$$
\begin{aligned}
\mathbf{x}(a(v), w) & =f(\mathbf{x}(v, w), \mathbf{u}(v, w), v, w) \\
0 & =h(\mathbf{x}(v, w), \mathbf{u}(v, w), v, w)
\end{aligned}
$$

if and only if the following linear equations are satisfied for $l=1,2, \cdots$,

$$
\begin{aligned}
X_{l w} A^{[l]} & =F_{100}(w) X_{l w}+F_{010}(w) U_{l w}+E_{l w} \\
0 & =H_{100}(w) X_{l w}+H_{010}(w) U_{l w}+F_{l w}
\end{aligned}
$$

where $A^{[l]}=M_{l} A_{1}^{(l)} N_{l}$, for $l=1,2, \cdots, E_{1 w}=F_{001}(w), F_{1 w}=H_{001}(w)$ and for $l=2,3, \cdots, k$,

$$
\begin{aligned}
E_{l w} & =\left(\sum_{n=2}^{l} \sum_{\substack{i+j+k=n \\
i, j, k \geq 0}} F_{i j k}(w) G_{l-n}^{i j}-\sum_{k=1}^{l-1} X_{k w} M_{k} \xi_{k, l}\right) N_{l} \\
F_{l w} & =\left(\sum_{n=2}^{l} \sum_{\substack{i+j+k=n \\
i, j, k \geq 0}} H_{i j k}(w) G_{l-n}^{i j}\right) N_{l}
\end{aligned}
$$


where

$$
\begin{aligned}
& \xi_{i, j}=\sum_{\substack{j_{1}+j_{2}+\cdots+j_{i}=j \\
j_{1}, j_{2}, \cdots, j_{i} \geq 1}} A_{j_{1}} \otimes A_{j_{2}} \otimes \cdots \otimes A_{j_{i}}, \quad i=1,2, \cdots, j \geq i \\
& G_{m}^{i j}=\left\{\begin{array}{cc}
0, & i=j=0, m>0 \\
1, & i=j=0, m=0 \\
\delta_{i, i+m}, & j=0, i=1,2, \cdots \\
\lambda_{j, j+m}, & i=0, j=1,2, \cdots \\
\sum_{k=0}^{m} \delta_{i, i+k} \otimes \lambda_{j, j+m-k}, & i, j=1,2, \cdots
\end{array}\right. \\
& \delta_{i, j}=\sum_{\substack{j_{1}+j_{2}+\cdots+j_{i}=j \\
j_{1}, j_{2}, \cdots, j_{i} \geq 1}} X_{j w_{1}} M_{j_{1}} \otimes X_{j w_{2}} M_{j_{2}} \otimes \cdots \otimes X_{j w_{i}} M_{j_{i}}, \\
& i=1,2, \cdots, j \geq i \\
& \lambda_{i, j}=\sum_{\substack{j_{1}+j_{2}+\cdots+j_{i}=j \\
j_{1}, j_{2}, \cdots, j_{i} \geq 1}} U_{j w_{1}} M_{j_{1}} \otimes U_{j w_{2}} M_{j_{2}} \otimes \cdots \otimes U_{j w_{i}} M_{j_{i}} \\
& i=1,2, \cdots, j \geq i .
\end{aligned}
$$

Proof. The proof of this lemma is similar to its continuous-time counterpart, Lemma 5.1 of [15], and is thus omitted here.

REMARK 2.5. Equations (24) are a set of Sylvester equations. They have a unique solution for any $E_{l w}$ and $F_{l w}$ if and only if Assumption $A 4$ holds [15]. In fact, it is shown that the eigenvalues of the matrix $A^{[l]}=M_{l} A_{1}^{(l)} N_{l}$ are given by

$$
\left\{\lambda \mid \lambda=\lambda_{1}^{l_{1}} \times \lambda_{2}^{l_{2}} \times \cdots \times \lambda_{q}^{l_{q}}, l_{1}+l_{2}+\cdots+l_{q}=l, \quad l_{1}, l_{2}, \cdots, l_{q}=0,1,2, \cdots\right\},
$$

where $\lambda_{1}, \lambda_{2}, \cdots, \lambda_{q}$ are the eigenvalues of the matrix $A_{1}$ [11]. Thus assumption $A 4$ simply says that the transmission zeros of the linearization of the given plant do not coincide with the eigenvalues of the matrix $A^{[l]}$ for all $l=1,2, \cdots$.

\section{Solvability of Discrete-time $k^{t h}$-Order Robust Servomechanism} Problem. Let

$$
\begin{aligned}
f(x, u, v, w) & =A(w) x+B(w) u+E(w) v+f_{2}(x, u, v, w) \\
h(x, u, v, w) & =C(w) x+D(w) u+F(w) v+h_{2}(x, u, v, w) \\
\phi(x, z) & =K_{1} x+K_{2} z+\phi_{2}(x, z) \\
g(z, e) & =G_{1} z+G_{2} e+g_{2}(z, e) \\
f_{c}\left(x_{c}, v, w\right) & =A_{c}(w) x_{c}+B_{c}(w) v+f_{c 2}\left(x_{c}, v, w\right) \\
a(v) & =A_{1} v+a_{2}(v)
\end{aligned}
$$


where

$$
\begin{aligned}
& A(w)=\frac{\partial f}{\partial x}(0,0,0, w), \quad B(w)=\frac{\partial f}{\partial u}(0,0,0, w), \quad E(w)=\frac{\partial f}{\partial v}(0,0,0, w) \\
& C(w)=\frac{\partial h}{\partial x}(0,0,0, w), \quad D(w)=\frac{\partial h}{\partial u}(0,0,0, w), \quad F(w)=\frac{\partial h}{\partial v}(0,0,0, w) \\
& K_{1}=\frac{\partial \phi}{\partial x}(0,0), \quad K_{2}=\frac{\partial \phi}{\partial z}(0,0), \quad G_{1}=\frac{\partial g}{\partial z}(0,0), \quad G_{2}=\frac{\partial g}{\partial e}(0,0) \\
& A_{c}(w)=\frac{\partial f_{c}}{\partial x}(0,0, w), \quad B_{c}(w)=\frac{\partial f_{c}}{\partial v}(0,0, w), \quad A_{1}=\frac{\partial a}{\partial v}(0) .
\end{aligned}
$$

For convenience, in the sequel, we will use the shorthand notation $A, B$, and so forth to denote $A(0), B(0)$, and so forth.

Now, assume a control law of the form (3) with $g(z, e)=G_{1} z+G_{2} e$ renders the closed-loop system (5) has property R1. Then by the center manifold theorem for map [2][11], there exists a locally defined sufficiently smooth function $\overline{\mathbf{x}}_{c}(v, w)$ with $\overline{\mathbf{x}}_{c}(0,0)=0$ such that, for $v \in V, w \in W$,

$$
\overline{\mathbf{x}}_{c}(a(v), w)=f_{c}\left(\overline{\mathbf{x}}_{c}(v, w), v, w\right)
$$

By partitioning $\overline{\mathbf{x}}_{c}(v, w)=(\overline{\mathbf{x}}(v, w), \overline{\mathbf{z}}(v, w)),(31)$ becomes

$$
\begin{aligned}
\overline{\mathbf{x}}(a(v), w) & =f(\overline{\mathbf{x}}(v, w), \phi(\overline{\mathbf{x}}(v, w), \overline{\mathbf{z}}(v, w)), v, w) \\
\overline{\mathbf{z}}(a(v), w) & =G_{1} \overline{\mathbf{x}}(v, w)+G_{2} \mathbf{e}(v, w)
\end{aligned}
$$

where

$$
\mathbf{e}(v, w)=h(\overline{\mathbf{x}}(v, w), \phi(\overline{\mathbf{x}}(v, w), \overline{\mathbf{z}}(v, w)), v, w) .
$$

Express $\overline{\mathbf{x}}(v, w), \overline{\mathbf{z}}(v, w)$, and $\mathbf{e}(v, w)$ uniquely as

$$
\begin{aligned}
& \overline{\mathbf{x}}(v, w)=\sum_{l=1}^{k} X_{l w} v^{[l]}+o^{k}(v) \\
& \overline{\mathbf{z}}(v, w)=\sum_{l=1}^{k} Z_{l w} v^{[l]}+o^{k}(v) \\
& \mathbf{e}(v, w)=\sum_{l=1}^{k} Y_{l w} v^{[l]}+o^{k}(v)
\end{aligned}
$$

where $\left(X_{l w}\right.$ and $\left.Z_{l w}\right)$ are constant matrices of appropriate dimensions depending perhaps on $w$. By Lemma 2.1, for $l=1,2, \cdots, k$,

$$
\begin{aligned}
X_{l w} A^{[l]} & =\bar{A}_{c}(w) X_{l w}+\bar{B}_{c}(w) Z_{l w}+E_{l w} \\
Z_{l w} A^{[l]} & =G_{1} Z_{l w}+G_{2}\left(\bar{C}_{c}(w) X_{l w}+\bar{D}_{c}(w) Z_{l w}+F_{l w}\right)
\end{aligned}
$$

and

$$
Y_{l w}=\bar{C}_{c}(w) X_{l w}+\bar{D}_{c}(w) Z_{l w}+F_{l w}
$$


where

$$
\begin{array}{ll}
\bar{A}_{c}(w)=A(w)+B(w) K_{1}, & \bar{B}_{c}(w)=B(w) K_{2} \\
\bar{C}_{c}(w)=C(w)+D(w) K_{1}, & \bar{D}_{c}(w)=D(w) K_{2}
\end{array}
$$

for $l=1, \cdots, k, A^{[l]}=M_{l} A_{1}^{(l)} N_{l},\left(E_{1 w}, F_{1 w}\right)=(E(w), F(w))$, and for $l=2,3, \cdots, k$, $\left(E_{l w}, F_{l w}\right)$ are described in (25) and (26).

Lemma 3.1. Under assumption A1, assume a control law of the form (3) with $g(z, e)=G_{1} z+G_{2}$ e renders the closed-loop system (5) property $R 1$. Then,

(i). $Y_{l w}=0$ for all $w \in W$ if the pair $\left(G_{1}, G_{2}\right)$ incorporates an internal model of the matrix $A^{[l]}$.

(ii). The $k^{\text {th }}$-order robust servomechanism problem is solved if the pair $\left(G_{1}, G_{2}\right)$ incorporates an internal model of the matrix $A_{k f}$ where

$$
A_{k f}=\left[\begin{array}{cccc}
A^{[1]} & 0 & \cdots & 0 \\
0 & A^{[2]} & \cdots & 0 \\
\vdots & \vdots & \vdots & \vdots \\
0 & 0 & \cdots & A^{[k]}
\end{array}\right] .
$$

Proof. (i) Since the closed-loop system (5) satisfies R1, the matrix

$$
A_{c}=\left[\begin{array}{cc}
A+B K_{1} & B K_{2} \\
G_{2}\left(C+D K_{1}\right) & G_{1}+G_{2} D K_{2}
\end{array}\right]
$$

is Schur. Under assumption A1, we have $\sigma\left(A^{[l]}\right) \bigcap \sigma\left(A_{c}\right)=\emptyset$. Thus, by Remark 2.4, $Y_{l w}=0$ for all $w \in W$ if the pair $\left(G_{1}, G_{2}\right)$ incorporates an internal model of the matrix $A^{[l]}$.

(ii) By definition of $A_{k f}$, if the pair $\left(G_{1}, G_{2}\right)$ incorporates an internal model of the matrix $A_{k f}$, it also incorporates an internal model of all the matrix $A^{[l]}$ for $l=1,2, \cdots, k$. Therefore, the control law renders $Y_{l w}=0$ for all $l=1,2, \cdots, k$. As a result, by Remark 2.1, the controller solves the $k^{t h}$-order robust servomechanism problem.

REMARK 3.1. It is interesting to know that if $v(t)$ satisfies $v(t+1)=A_{1} v(t)$, then we have $v^{[l]}(t+1)=A^{[l]} v^{[l]}$. Let

$$
v_{k f}=\left[\begin{array}{c}
v^{[1]} \\
v^{[2]} \\
\vdots \\
v^{[k]}
\end{array}\right]
$$

Then the matrix $A_{k f}$ is such that

$$
v_{k f}(t+1)=A_{k f} v_{k f} .
$$


The system (39) can be considered as a generalized exosystem which not only generates the exogenous signal $v$ (when $a(v)=A_{1} v$ ), but also the higher order terms of the exogenous signal $v$ up to order $k$. The system (39) is called a $k$-fold exosystem. Lemma 3.1 asserts that designing a discrete-time $k^{\text {th }}$-order robust controller for a nonlinear system (1) is equivalent to designing a linear discrete-time robust controller for the linear system consisting of the linear approximation of (1) and the $k$-fold exosystem (39).

As pointed out in Remark 2.3, under assumption A4, it is always possible to find two matrices $G_{1}$ and $G_{2}$ such that they incorporate an internal model of the matrix $A_{k f}$. Thus we can define an augmented system as follows

$$
\begin{aligned}
x(t+1) & =f(x(t), u(t), v(t), w) \\
z(t+1) & =G_{1} z(t)+G_{2} e(t) \\
e(t) & =h(x(t), u(t), v(t), w)
\end{aligned}
$$

where the pair $\left(G_{1}, G_{2}\right)$ incorporates an internal model of the matrix $A_{k f}$. By Lemma 3.1 , the discrete-time $k^{t h}$-order robust servomechanism problem is solvable by a control law of the form (3) with $g(z, e)=G_{1} z+G_{2} e$ if the state feedback control law of the form $u=\phi(x, z)$ can exponentially stabilize the equilibrium point of the augmented system (40). Thus we have the following result.

THEOREM 3.1. (i) Under assumptions A1, A2, and A4, for any positive integer $k$, let $\left(G_{1}, G_{2}\right)$ incorporate an internal model of the matrix $A_{k f}$, then there exist feedback gains $K_{1}$ and $K_{2}$ such that the discrete-time $k^{\text {th }}$-order robust servomechanism problem is solvable by a linear state feedback control of the form

$$
\begin{aligned}
u(t) & =K_{1} x(t)+K_{2} z(t) \\
z(t+1) & =G_{1} z(t)+G_{2} e(t) .
\end{aligned}
$$

(ii) Under assumptions $A 1$ to $A 4$, for any positive integer $k$, let $\left(\mathcal{G}_{1}, \mathcal{G}_{2}\right)$ incorporate an internal model of the matrix $A_{k f}$, then there exists a feedback gain $K$ such that the discrete-time $k^{\text {th }}$-order robust servomechanism problem is solvable by a linear output feedback control of the form

$$
\begin{aligned}
u(t) & =K z(t) \\
z(t+1) & =\mathcal{G}_{1} z(t)+\mathcal{G}_{2} e(t) .
\end{aligned}
$$

Proof. Part (i). The eigenvalues of the matrix $A^{[l]}$ are given by

$$
\left\{\lambda \mid \lambda=\lambda_{1}^{l_{1}} \times \cdots \times \lambda_{q}^{l_{q}}, l_{1}+\cdots+l_{q}=l, l_{1}, \cdots, l_{q}=0,1,2, \cdots\right\}
$$

where $\lambda_{1}, \cdots, \lambda_{q}$ are eigenvalues of $A_{1}$. Under assumption A4, there exist $\left(G_{1}, G_{2}\right)$ that incorporates an internal model of $A_{k f}$ for any integer $k>0$. Therefore, by 
Remark 2.4, the pair

$$
\left[\begin{array}{cc}
A & 0 \\
G_{2} C & G_{1}
\end{array}\right], \quad\left[\begin{array}{c}
B \\
G_{2} D
\end{array}\right]
$$

is stabilizable because of assumption A2. Thus there exist feedback gains $K_{1}$ and $K_{2}$ such that the matrix

$$
\left[\begin{array}{cc}
A+B K_{1} & B K_{2} \\
G_{2}\left(C+D K_{1}\right) & G_{1}+G_{2} D K_{2}
\end{array}\right]
$$

is Schur, i.e., under the control law (41), the closed-loop system satisfies R1. With assumption A1, it follows from part (ii) of Lemma 3.1 that the control law (41) solves the discrete-time $k^{\text {th }}$-order robust servomechanism problem.

Part (ii). Let $\left(K_{1}, K_{2}, G_{1}, G_{2}\right)$ be what has been obtained from part (i). Under assumption A3, there exists $L$ such that $A-L C$ is Schur. Let $K=\left(K_{1}, K_{2}\right)$,

$$
\mathcal{G}_{1}=\left[\begin{array}{cc}
A+B K_{1}-L\left(C+D K_{1}\right) & (B-L D) K_{2} \\
0 & G_{1}
\end{array}\right], \quad \mathcal{G}_{2}=\left[\begin{array}{c}
L \\
G_{2}
\end{array}\right] .
$$

Clearly, the pair $\left(\mathcal{G}_{1}, \mathcal{G}_{2}\right)$ incorporates an internal model of the matrix $A_{k f}$. Moreover, under the control law (42), the Jacobian matrix of the closed-loop system is given by

$$
\left[\begin{array}{ccc}
A & B K_{1} & B K_{2} \\
L C & A+B K_{1}-L C & B K_{2} \\
G_{2} C & G_{2} D K_{1} & G_{1}+G_{2} D K_{2}
\end{array}\right]
$$

Subtracting the first row from the second row and then adding the second column to the first column shows that the spectrum of (45) is given by those of (44) and $A-L C$. Thus, the closed-loop system satisfies R1. Again, it follows from Lemma 3.1 that the control law (42) solves the discrete-time $k^{\text {th }}$-order robust servomechanism problem.

REMARK 3.2. The solvability condition of the discrete-time $k^{\text {th }}$-order robust servomechanism problem is exactly the same as that of the discrete-time $k^{\text {th }}$-order approximate servomechanism problem studied in [14], [21]. But the design philosophy of the control laws are completely different. The controller that solves the latter problem relies on the approximation solution of the regulator equations thus demanding the complete knowledge of the plant. On the other hand, the $k^{\text {th }}$-order robust controller is designed completely based on the linearization of the given nonlinear plant at the origin. Regardless of the variations of the uncertain parameter $w$, the controller can guarantee the zero steady state tracking error up to the order $k$ of the exogenous signal $v$.

4. Solvability of Discrete-time Robust Nonlinear Servomechanism Problem. In this section, we will further show that, under some additional assumptions on the solution of the discrete regulator equations, a control law solving the 
discrete-time $k^{\text {th }}$-order robust servomechanism problem for the composite system (1) and (2) also solves the discrete-time robust servomechanism problem for the same system.

Lemma 4.1. Under assumption A1, assume a control law of the form (3) is such that the closed-loop system satisfies $R 1$, if there exist sufficiently smooth functions $(\mathbf{x}(v, w), \mathbf{u}(v, w), \mathbf{z}(v, w))$ locally defined in $v \in V, w \in W$ with $(\mathbf{x}(0,0), \mathbf{u}(0,0)$, $\mathbf{z}(0,0))=(0,0,0)$ such that $\mathbf{x}(v, w)$ and $\mathbf{u}(v, w)$ are the solution of the discrete regulator equations (15), and $\mathbf{z}(v, w)$ satisfies

$$
\begin{aligned}
\mathbf{u}(v, w) & =\phi(\mathbf{x}(v, w), \mathbf{z}(v, w)) \\
\mathbf{z}(a(v), w) & =g(\mathbf{z}(v, w), 0)
\end{aligned}
$$

then the control law also solves the robust servomechanism problem.

Proof. By Remark 2.1, we only need to show that there exists a sufficiently smooth function $\mathbf{x}_{c}(v, w)$ with $\mathbf{x}_{c}(0,0)=0$ that satisfies (11) and (12). To this end, define $\mathbf{x}_{c}(v, w)=(\mathbf{x}(v, w), \mathbf{z}(v, w))$. Using (6) yields

$$
\begin{aligned}
& h_{c}\left(\mathbf{x}_{c}(v, w), v, w\right)=h(\mathbf{x}(v, w), \phi(\mathbf{x}(v, w), \mathbf{z}(v, w)), v, w) \\
& f_{c}\left(\mathbf{x}_{c}(v, w), v, w\right)=\left[\begin{array}{c}
f(\mathbf{x}(v, w), \phi(\mathbf{x}(v, w), \mathbf{z}(v, w)), v, w) \\
g\left(\mathbf{z}(v, w), h_{c}\left(\mathbf{x}_{c}(v, w), v, w\right)\right)
\end{array}\right]
\end{aligned}
$$

Substituting (46) into (48) and (49) gives

$$
\begin{aligned}
h_{c}\left(\mathbf{x}_{c}(v, w), v, w\right) & =h(\mathbf{x}(v, w), \mathbf{u}(v, w), v, w) \\
f_{c}\left(\mathbf{x}_{c}(v, w), v, w\right) & =\left[\begin{array}{c}
f(\mathbf{x}(v, w), \mathbf{u}(v, w), v, w) \\
g\left(\mathbf{z}(v, w), h_{c}\left(\mathbf{x}_{c}(v, w), v, w\right)\right)
\end{array}\right]
\end{aligned}
$$

Using equation (15) and equation (47) in (50) and (51) gives

$$
\begin{aligned}
h_{c}\left(\mathbf{x}_{c}(v, w), v, w\right) & =h(\mathbf{x}(v, w), \mathbf{u}(v, w), v, w)=0, \\
f_{c}\left(\mathbf{x}_{c}(v, w), v, w\right) & =\left[\begin{array}{c}
f(\mathbf{x}(v, w), \mathbf{u}(v, w), v, w) \\
g\left(\mathbf{z}(v, w), h_{c}\left(\mathbf{x}_{c}(v, w), v, w\right)\right)
\end{array}\right] \\
& =\left[\begin{array}{c}
\mathbf{x}(a(v), w) \\
g(\mathbf{z}(v, w), 0)
\end{array}\right]=\left[\begin{array}{l}
\mathbf{x}(a(v), w) \\
\mathbf{z}(a(v), w)
\end{array}\right]=\mathbf{x}_{c}(a(v), w) .
\end{aligned}
$$

To solve the robust servomechanism problem, we need to impose an additional assumption on the exosystem (2).

A6: $a(v)=A_{1} v$ for some matrix $A_{1}$, and all the eigenvalues of $A_{1}$ are simple and lie on the unit circle.

TheOrem 4.1. (i) Under Assumptions A2, A4, A5 and A6, assume the solution $\mathbf{x}(v, w)$ and $\mathbf{u}(v, w)$ of the discrete regulator equations (15) are $k^{\text {th }}$ degree polynomials 
in $v$. Then if the state feedback controller (41) solves the discrete-time $k^{\text {th }}$-order robust servomechanism problem, it also solves the discrete-time robust servomechanism problem.

(ii) Under assumptions A2-A6, assume the solution $\mathbf{u}(v, w)$ of the discrete regulator equations (15) is a $k^{\text {th }}$ degree polynomial in $v$. Then if the output feedback controller (42) solves the discrete-time $k^{\text {th }}$-order robust servomechanism problem, it also solves the discrete-time robust servomechanism problem.

Proof. Part(i). Assume the controller (41) solves the discrete-time $k^{\text {th }}$-order robust servomechanism problem. By Lemma 4.1, it suffices to show that there exists a sufficiently smooth function $\mathbf{z}(v, w)$ such that

$$
\begin{aligned}
\mathbf{u}(v, w) & =K_{1} \mathbf{x}(v, w)+K_{2} \mathbf{z}(v, w) \\
\mathbf{z}\left(A_{1} v, w\right) & =G_{1} \mathbf{z}(v, w) .
\end{aligned}
$$

To this end, let $\overline{\mathbf{x}}(v, w)$ and $\overline{\mathbf{z}}(v, w)$ be sufficiently smooth functions satisfying (32), and $\mathbf{e}(v, w)$ be as defined in (33). Again, express $\overline{\mathbf{x}}(v, w), \overline{\mathbf{z}}(v, w)$ and $\mathbf{e}(v, w)$ as in (34). Since the controller (41) solves the discrete-time $k^{\text {th }}$-order robust servomechanism problem, for $l=1, \cdots, k, X_{l w}$ and $Z_{l w}$ satisfy (35) and (36) with $Y_{l w}=0$ where

$$
\begin{array}{ll}
\bar{A}_{c}(w)=A(w)+B(w) K_{1}, & \bar{B}_{c}(w)=B(w) K_{2} \\
\bar{C}_{c}(w)=C(w)+D(w) K_{1}, & \bar{D}_{c}(w)=D(w) K_{2} .
\end{array}
$$

Let $U_{l w}=K_{1} X_{l w}+K_{2} Z_{l w}$. Then (35) and (36) imply, for $l=1, \cdots, k$,

$$
\begin{aligned}
X_{l w} A^{[l]} & =A(w) X_{l w}+B(w) U_{l w}+E_{l w} \\
0 & =C(w) X_{l w}+D(w) U_{l w}+F_{l w} .
\end{aligned}
$$

By Lemma 2.1, there exist sufficiently smooth functions $\mathbf{x}_{k}(v, w)=o^{k}(v)$ and $\mathbf{u}_{k}(v, w)$ $=o^{k}(v)$ such that

$$
\begin{aligned}
& \mathbf{x}(v, w)=\sum_{l=1}^{k} X_{l w} v^{[l]}+\mathbf{x}_{k}(v, w) \\
& \mathbf{u}(v, w)=\sum_{l=1}^{k} U_{l w} v^{[l]}+\mathbf{u}_{k}(v, w) .
\end{aligned}
$$

However, by assumption of this theorem, $\mathbf{x}(v, w)$ and $\mathbf{u}(v, w)$ are $k^{t h}$ degree polynomials in $v$, thus

$$
\begin{aligned}
& \mathbf{x}(v, w)=\sum_{l=1}^{k} X_{l w} v^{[l]} \\
& \mathbf{u}(v, w)=\sum_{l=1}^{k} U_{l w} v^{[l]}
\end{aligned}
$$


Let

$$
\mathbf{z}(v, w)=\sum_{l=1}^{k} Z_{l w} v^{[l]} .
$$

Clearly, (52) is satisfied. Now using (35) and (36) yields

$$
Z_{l w} A^{[l]}=G_{1} Z_{l w}, \quad l=1,2, \cdots, k .
$$

Multiplying (54) from the right by $v^{[l]}$ and then summarizing from $l=1$ to $k$ gives

$$
\sum_{l=1}^{k} Z_{l w} A^{[l]} v^{[l]}=\sum_{l=1}^{k} G_{1} Z_{l w} v^{[l]} .
$$

Thus,

$$
\begin{aligned}
\mathbf{z}\left(A_{1} v, w\right) & =\sum_{l=1}^{k} Z_{l w}\left(A_{1} v\right)^{[l]}=\sum_{l=1}^{k} Z_{l w} M_{l}\left(A_{1} v\right)^{(l)} \\
& =\sum_{l=1}^{k} Z_{l w} M_{l} A_{1}^{(l)} N_{l} v^{[l]}=\sum_{l=1}^{k} Z_{l w} A^{[l]} v^{[l]} \\
& =\sum_{l=1}^{k} G_{1} Z_{l w} v^{[l]}=G_{1} \mathbf{z}(v, w) .
\end{aligned}
$$

Part (ii). The proof of part (ii) is almost the same as that of part (i). Assume a controller of the form (42) solves the discrete-time $k^{\text {th }}$-order robust servomechanism problem. By Lemma 4.1, we need to show the existence of a sufficiently smooth function $\mathbf{z}(v, w)$ with $\mathbf{z}(0,0)=0$ that satisfies

$$
\begin{aligned}
\mathbf{u}(v, w) & =K \mathbf{z}(v, w) \\
\mathbf{z}\left(A_{1} v, w\right) & =\mathcal{G}_{1} \mathbf{z}(v, w) .
\end{aligned}
$$

Let $\overline{\mathbf{x}}(v, w)$ and $\overline{\mathbf{z}}(v, w)$ be sufficiently smooth functions satisfying (32), and $\mathbf{e}(v, w)$ be as defined in (33). Again, express $\overline{\mathbf{x}}(v, w), \overline{\mathbf{z}}(v, w)$ and $\mathbf{e}(v, w)$ as in (34). Since the controller (42) solves discrete-time $k^{\text {th }}$-order robust servomechanism problem, for $l=1, \cdots, k, X_{l w}$ and $Z_{l w}$ satisfy (35) and (36) with $Y_{l w}=0$ where

$$
\begin{array}{ll}
\bar{A}_{c}(w)=A(w), & \bar{B}_{c}(w)=B(w) K \\
\bar{C}_{c}(w)=C(w), & \bar{D}_{c}(w)=D(w) K .
\end{array}
$$

Let $U_{l w}=K Z_{l w}$, then (35) and (36) imply, for $l=1, \cdots, k$,

$$
\begin{aligned}
X_{l w} A^{[l]} & =A(w) X_{l w}+B(w) U_{l w}+E_{l w} \\
0 & =C(w) X_{l w}+D(w) U_{l w}+F_{l w} .
\end{aligned}
$$


By Lemma 2.1, there exist sufficiently smooth functions $\mathbf{x}_{k}(v, w)=o^{k}(v)$ and $\mathbf{u}_{k}(v, w)$ $=o^{k}(v)$ such that

$$
\begin{aligned}
& \mathbf{x}(v, w)=\sum_{l=1}^{k} X_{l w} v^{[l]}+\mathbf{x}_{k}(v, w) \\
& \mathbf{u}(v, w)=\sum_{l=1}^{k} U_{l w} v^{[l]}+\mathbf{u}_{k}(v, w) .
\end{aligned}
$$

However, by assumption of this theorem, $\mathbf{u}(v, w)$ is $k^{\text {th }}$ degree polynomials in $v$, thus

$$
\mathbf{u}(v, w)=\sum_{l=1}^{k} U_{l w} v^{[l]} .
$$

Let

$$
\mathbf{z}(v, w)=\sum_{l=1}^{k} Z_{l w} v^{[l]} .
$$

Clearly, (56) is satisfied. The proof of satisfaction of (57) is the same as that (53) in part (i), thus is omitted.

REMARK 4.1. If the exogenous signal $v$ is available for control, it is possible to somehow relax the restriction on $\mathbf{x}(v, w)$ and $\mathbf{u}(v, w)$ as shown by the following theorem.

TheOREm 4.2. (i). Under assumptions A2, A4, A5 and A6, and suppose that there exists some integer $k>0$ such that $\mathbf{x}(v, w)$ and $\mathbf{u}(v, w)$ take the following form

$$
\begin{aligned}
& \mathbf{x}(v, w)=\mathbf{x}^{[k]}(v, w)+\mathbf{x}_{h k}(v) \\
& \mathbf{u}(v, w)=\mathbf{u}^{[k]}(v, w)+\mathbf{u}_{h k}(v) .
\end{aligned}
$$

where $\mathbf{x}^{[k]}(v, w)$ and $\mathbf{u}^{[k]}(v, w)$ are degree $k$ polynomials of $v$ with coefficients depending on $w$, and $\mathbf{x}_{h k}(v)$ and $\mathbf{u}_{h k}(v)$ are some sufficiently smooth function of $v$, independent of $w$, vanishing at the origin together with their derivatives up to order $k$. If the state feedback controller (41) solves the discrete-time $k^{\text {th }}$-order robust servomechanism problem, then the following controller

$$
\begin{aligned}
u(t) & =K_{1}\left(x(t)-\mathbf{x}_{h k}(v(t))\right)+K_{2} z(t)+\mathbf{u}_{h k}(v(t)) \\
z(t+1) & =G_{1} z(t)+G_{2} e(t)
\end{aligned}
$$

solves the discrete-time robust servomechanism problem.

(ii). Under assumptions A2 to A6, and suppose that there exists some integer $k>0$ such that $\mathbf{u}(v, w)$ takes the form of

$$
\mathbf{u}(v, w)=\mathbf{u}^{[k]}(v, w)+\mathbf{u}_{h k}(v) .
$$


If the output feedback controller (42) solves the discrete-time $k^{\text {th }}$-order robust servomechanism problem, then the following controller

$$
\begin{aligned}
u(t) & =K z(t)+\mathbf{u}_{h k}(v(t)) \\
z(t+1) & =\mathcal{G}_{1} z(t)+\mathcal{G}_{2} e(t)
\end{aligned}
$$

solves the discrete-time robust servomechanism problem.

Proof. Part (i). Applying a state and input transformation $x=x^{\prime}+\mathbf{x}_{h k}(v)$, $u=u^{\prime}+\mathbf{u}_{h k}(v)$ to (1) gives

$$
\begin{aligned}
x^{\prime}(t+1) & =f\left(x^{\prime}(t)+\mathbf{x}_{h k}(v(t)), u^{\prime}(t)+\mathbf{u}_{h k}(v(t)), v(t), w\right)-\mathbf{x}_{h k}\left(A_{1} v(t)\right) \\
e(t) & =h\left(x^{\prime}(t)+\mathbf{x}_{h k}(v(t)), u^{\prime}(t)+\mathbf{u}_{h k}(v(t)), v(t), w\right) .
\end{aligned}
$$

It can be verified that $\mathbf{x}^{\prime}(v, w)=\mathbf{x}(v, w)-\mathbf{x}_{h k}(v)$ and $\mathbf{u}^{\prime}(v, w)=\mathbf{u}(v, w)-\mathbf{u}_{h k}(v)$ are the solution of the discrete regulator equations associated with the system (60). It is clear that the system (60) still satisfies assumptions A2, A4, A5 and A6, and $\mathbf{x}^{\prime}(v, w)$ and $\mathbf{u}^{\prime}(v, w)$ are degree $k$ polynomials in $v$, by Theorem 4.1, there exists a state feedback controller of the form (41) that solves the robust output regulation problem for system (60). Thus, a controller of the form (58) solves the robust output regulation problem for system (1).

Part (ii). The proof of Part (ii) is almost the same as the proof of Part (i), and is thus omitted.

5. Example. In this section, we will consider an asymptotic tracking problem for the inverted pendulum on a cart system. The state space model of the inverted pendulum on a cart system is given in [9],

$$
\begin{aligned}
\dot{x}_{1} & =x_{2} \\
\dot{x}_{2} & =\frac{1}{M+m\left(\sin x_{3}\right)^{2}}\left(u+m l x_{4}^{2} \sin x_{3}-b x_{2}-m g \cos n x_{3} \sin x_{3}\right) \\
\dot{x}_{3} & =x_{4} \\
\dot{x}_{4} & =\frac{1}{l\left(M+m\left(\sin x_{3}\right)^{2}\right)}\left((M+m) g \sin x_{3}-u \cos x_{3}+b x_{2} \cos x_{3}\right. \\
& \left.\quad-m l x_{4}^{2} \cos x_{3} \sin x_{3}\right)
\end{aligned}
$$

where $M$ is the mass of the cart, $m$ the mass of the block on the pendulum, $l$ the length of the pendulum, $g$ the acceleration due to gravity, $b$ the coefficient of viscous friction for motion of the cart, $u$ the applied force. The four state variables stand for $x_{1}=x, x_{2}=\dot{x}, x_{3}=\theta, x_{4}=\dot{\theta}$ where $x$ is the position of the cart, and $\theta$ is the angle the pendulum makes with vertical, as shown in Figure 1. The discrete-time model is obtained by discretizing the continuous time model (61) via Euler's method with $T$ 
as the sampling period [21],

$$
\begin{aligned}
x_{1}(t+1)= & x_{1}(t)+T x_{2}(t) \\
x_{2}(t+1)= & x_{2}(t)+\frac{T}{M+m\left(\sin x_{3}(t)\right)^{2}}\left(u(t)+m l x_{4}^{2}(t) \sin x_{3}(t)\right. \\
& \left.-b x_{2}(t)-m g \cos x_{3}(t) \sin x_{3}(t)\right) \\
x_{3}(t+1)= & x_{3}(t)+T x_{4}(t) \\
x_{4}(t+1)= & x_{4}(t)+\frac{T}{l\left(M+m\left(\sin x_{3}(t)\right)^{2}\right)}\left((M+m) g \sin x_{3}(t)-u(t) \cos x_{3}(t)\right. \\
& \left.+b x_{2}(t) \cos x_{3}(t)-m l x_{4}^{2}(t) \cos x_{3}(t) \sin x_{3}(t)\right) \\
y(t)= & x_{1}(t)
\end{aligned}
$$

We will consider the asymptotic tracking of the output $y(t)$ to a sinusoidal function $y_{d}(t)=A_{m} \sin (\omega T t)$. Thus the exosystem is given by

$$
v(t+1)=\alpha(v(t))=A_{1} v(t)
$$

with

$$
A_{1}=\left[\begin{array}{cc}
\cos \omega T & \sin \omega T \\
-\sin \omega T & \cos \omega T
\end{array}\right], \quad v(t)=\left[\begin{array}{c}
v_{1}(t) \\
v_{2}(t)
\end{array}\right], \quad v(0)=\left[\begin{array}{c}
0 \\
A_{m}
\end{array}\right] .
$$

It is clear that $v_{1}(t)=A_{m} \sin (\omega T t)$. Thus, we can define the error equation as follows

$$
e(t)=y(t)-v_{1}(t)=x_{1}(t)-v_{1}(t)
$$

The Jacobian linearization of the discrete-time inverted pendulum on a cart system (62) can be calculated as follows

$$
\begin{gathered}
A=\frac{\partial f(0,0,0,0)}{\partial x}=\left[\begin{array}{cccc}
1 & T & 0 & 0 \\
0 & 1-\frac{b T}{M} & -\frac{m g T}{M} & 0 \\
0 & 0 & 1 & T \\
0 & \frac{b T}{l M} & \frac{(M+m) g T}{l M} & 1
\end{array}\right], B=\frac{\partial f(0,0,0,0)}{\partial u}=\left[\begin{array}{c}
0 \\
\frac{T}{M} \\
0 \\
-\frac{T}{l M}
\end{array}\right] \\
C=\frac{\partial h(0,0,0,0)}{\partial x}=\left[\begin{array}{llll}
1 & 0 & 0 & 0
\end{array}\right],
\end{gathered}
$$

It is easy to verify that this system satisfies assumption A2 and A4. Thus, both the discrete-time $k^{t h}$-order approximate servomechanism problem and the discretetime $k^{t h}$-order robust servomechanism problem are solvable by state feedback. For comparison, we will design the third order approximate state feedback controller and the third order robust state feedback control for this system.

Third order approximate state feedback controller. The discrete regulator equations associated with the tracking control problem for the inverted pendulum on 
a cart system are

$$
\begin{aligned}
\mathbf{x}_{1}\left(A_{1} v\right)= & \mathbf{x}_{1}(v)+T \mathbf{x}_{2}(v) \\
\mathbf{x}_{2}\left(A_{1} v\right)= & \mathbf{x}_{2}(v)+\frac{T}{M+m\left(\sin \mathbf{x}_{3}(v)\right)^{2}}\left(\mathbf{u}(v)+m l \mathbf{x}_{4}^{2}(v) \sin \mathbf{x}_{3}(v)\right. \\
\mathbf{x}_{3}\left(A_{1} v\right)= & \left.\quad-b \mathbf{x}_{2}(v)-m g \cos \mathbf{x}_{3}(v) \sin \mathbf{x}_{3}(v)\right) \\
\mathbf{x}_{4}\left(A_{1} v\right)= & \mathbf{x}_{4}(v)+\frac{T}{l\left(M+m\left(\sin \mathbf{x}_{3}(v)\right)^{2}\right)}\left((M+m) g \sin \mathbf{x}_{3}(v)\right. \\
& -\mathbf{u}(v) \cos \mathbf{x}_{3}(v)+b \mathbf{x}_{2}(v) \cos \mathbf{x}_{3}(v) \\
& \left.-m l \mathbf{x}_{4}^{2}(v) \sin \mathbf{x}_{3}(v) \cos \mathbf{x}_{3}(v)\right) \\
0= & \mathbf{x}_{1}(v)-v_{1} .
\end{aligned}
$$

As shown in [21], (64) can be partially solved as follows,

$$
\begin{aligned}
& \mathbf{x}_{1}(v)=v_{1} \\
& \mathbf{x}_{2}(v)=\left(v_{1}(\cos \omega T-1)+v_{2} \sin \omega T\right) / T \\
& \mathbf{x}_{4}(v)=\left(\mathbf{x}_{3}\left(A_{1} v\right)-\mathbf{x}_{3}(v)\right) / T \\
& \mathbf{u}(v)=\frac{M+m\left(\sin \mathbf{x}_{3}(v)\right)^{2}}{T^{2}}\left[\begin{array}{ll}
\cos \omega T-1 & \sin \omega T
\end{array}\right]\left(A_{1}-I\right) v \\
& -m l \mathbf{x}_{4}^{2}(v) \sin \mathbf{x}_{3}(v)+\frac{b}{T}[\cos \omega T-1 \sin \omega T] v \\
& +m g \cos \mathbf{x}_{3}(v) \sin \mathbf{x}_{3}(v)
\end{aligned}
$$

with $\mathbf{x}_{3}(v)$ satisfying the following equation

$$
\begin{aligned}
\mathbf{x}_{3}\left(A_{1}^{2} v\right)=2 \mathbf{x}_{3}\left(A_{1} v\right)-\mathbf{x}_{3}(v)+\frac{g T^{2}}{l} \sin \mathbf{x}_{3}(v) \\
+\frac{\cos \mathbf{x}_{3}(v)}{l}\left[\begin{array}{ll}
\cos \omega T-1 & \sin \omega T]\left(I-A_{1}\right) v
\end{array}\right.
\end{aligned}
$$

where $I$ is the identity matrix. Therefore, as long as we can find out the function $\mathbf{x}_{3}(v)$ by solving $(69)$, we can then obtain $\mathbf{x}_{1}(v), \mathbf{x}_{2}(v), \mathbf{x}_{4}(v)$ and $\mathbf{u}(v)$ through $(65)$ to $(68)$.

Denote a third-order polynomial approximation for $\mathbf{x}_{3}(v)$ by $\mathbf{x}_{3}^{(3)}(v)$,

$$
\mathbf{x}_{3}^{(3)}(v)=a_{10} v_{1}+a_{01} v_{2}+a_{30} v_{1}^{3}+a_{21} v_{1}^{2} v_{2}+a_{12} v_{1} v_{2}^{2}+a_{03} v_{2}^{3} \text {. }
$$

Substituting (70) into (69) gives the coefficients of $\mathbf{x}_{3}^{(3)}(v)$ as follows:

$$
\begin{aligned}
& a_{10}=\frac{b_{1} b_{3}-b_{2} b_{4}}{b_{3}^{2}+b_{4}^{2}} \\
& a_{01}=\frac{b_{1} b_{4}+b_{2} b_{3}}{b_{3}^{2}+b_{4}^{2}}
\end{aligned}
$$

where

$$
\begin{array}{ll}
b_{1}=\left(\sin ^{2}(\omega T)-(1-\cos (\omega T))^{2}\right) / l, & b_{2}=2(1-\cos (\omega T)) \sin (\omega T) \\
b_{3}=\cos (2 \omega T)-2 \cos (\omega T)+1-g T^{2} / l, & b_{4}=2 \sin (\omega T)-\sin (2 \omega T)
\end{array}
$$


and

$$
\left[\begin{array}{l}
a_{30} \\
a_{21} \\
a_{12} \\
a_{03}
\end{array}\right]=\left[\begin{array}{cccc}
x_{11} & x_{12} & x_{13} & x_{14} \\
x_{21} & x_{22} & x_{23} & x_{24} \\
x_{24} & -x_{23} & x_{22} & -x_{21} \\
-x_{14} & x_{13} & -x_{12} & x_{11}
\end{array}\right]^{-1}\left[\begin{array}{c}
-\frac{g T^{2}}{6 l} a_{10}^{3}-\frac{1}{2} a_{10}^{2} b_{1} \\
-\frac{g T^{2}}{2 l} a_{10}^{2} a_{01}-\frac{1}{2}\left(2 a_{10} a_{01} b_{1}+a_{10}^{2} b_{2}\right) \\
-\frac{g T^{2}}{2 l} a_{10} a_{01}^{2}-\frac{1}{2}\left(2 a_{10} a_{01} b_{2}+a_{01}^{2} b_{1}\right) \\
-\frac{g T^{2}}{6 l} a_{01}^{3}-\frac{1}{2} a_{01}^{2} b_{2}
\end{array}\right]
$$

where

$$
\begin{aligned}
x_{11}= & \cos ^{3}(2 \omega T)-2 \cos ^{3}(\omega T)+1-g T^{2} / l, \\
x_{12}= & -\cos ^{2}(2 \omega T) \sin (2 \omega T)+2 \cos ^{2}(\omega T) \sin (\omega T), \\
x_{13}= & \cos (2 \omega T) \sin ^{2}(2 \omega T)-2 \cos (\omega T) \sin ^{2}(\omega T), \\
x_{14}= & -\sin ^{3}(2 \omega T)+2 \sin ^{3}(\omega T), \\
x_{21}= & 3 \cos ^{2}(2 \omega T) \sin (2 \omega T)-6 \cos ^{2}(\omega T) \sin (\omega T), \\
x_{22}= & \left(\cos ^{3}(2 \omega T)-2 \cos (2 \omega T) \sin ^{2}(2 \omega T)\right)-2\left(\cos ^{3}(\omega T)-2 \cos (\omega T) \sin ^{2}(\omega T)\right) \\
& +1-g T^{2} / l, \\
x_{23}= & \left(\sin ^{3}(2 \omega T)-2 \cos ^{2}(2 \omega T) \sin (2 \omega T)\right)-2\left(\sin ^{3}(\omega T)-2 \cos ^{2}(\omega T) \sin (\omega T)\right), \\
x_{24}= & 3 \sin ^{2}(2 \omega T) \cos (2 \omega T)-6 \sin ^{2}(\omega T) \cos (\omega T) .
\end{aligned}
$$

For example, when $\omega=0.5 \pi, g=9.8, l=0.325$ and $T=0.1$,

$$
\mathbf{x}_{3}^{(3)}(v)=-0.2300 v_{1}-0.0337 v_{2}+0.0039 v_{1}^{3}+0.001 v_{1}^{2} v_{2}+0.0012 v_{1} v_{2}^{2}+0.0003 v_{2}^{3},
$$

and in the case of $\omega=\pi, g=9.8, l=0.325$ and $T=0.1$,

$$
\mathbf{x}_{3}^{(3)}(v)=-0.7396 v_{1}-0.1792 v_{2}+0.1362 v_{1}^{3}+0.0292 v_{1}^{2} v_{2}+0.0734 v_{1} v_{2}^{2}+0.0221 v_{2}^{3} \text {. }
$$

With $\mathbf{x}_{3}^{(3)}(v)$ at hand, we can obtain the third order approximations of $\mathbf{x}(v)$ and $\mathbf{u}(v)$, denoted by $\mathbf{x}^{(3)}(v)$ and $\mathbf{u}^{(3)}(v)$, by using (65), (66), (67), and (68). Thus a third order state feedback controller is given as follows

$$
u(t)=\mathbf{u}^{(3)}(v(t))+K\left(x(t)-\mathbf{x}^{(3)}(v(t))\right)
$$

where the feedback gain $K$ is selected such that the eigenvalues of the matrix $A+B K$ are

$$
0.7488 \pm 0.4072 j, \quad 0.7679 \pm 0.1301 j
$$

which are obtained by a bilinear transformation from the ITAE prototype design for the continuous-time systems with the cutoff frequency equal to $4.0 \mathrm{rad} / \mathrm{sec}$.

Third order robust state feedback controller. To design a third order robust state feedback controller, we need to find a pair of matrices $\left(G_{1}, G_{2}\right)$ that incorporates an internal model of $A_{3 f}$. Since the solution of the discrete regulator equations does not contain the second order term, the output equation of the closed-loop system for any state feedback control law of the form (3) will not contain second order term 
either. Thus, it suffices to find a pair of matrices $\left(G_{1}, G_{2}\right)$ that incorporates an internal model of $A^{[1]}$ and $A^{[3]}$. The minimal polynomials of $A^{[1]}$ and $A^{[3]}$ are computed as follows:

$$
\begin{aligned}
& \alpha_{1}(\lambda)=\left(\lambda-e^{j \omega T}\right)\left(\lambda-e^{-j \omega T}\right) \\
& \alpha_{3}(\lambda)=\left(\lambda-e^{j \omega T}\right)\left(\lambda-e^{-j \omega T}\right)\left(\lambda-e^{j 3 \omega T}\right)\left(\lambda-e^{-j 3 \omega T}\right) .
\end{aligned}
$$

Thus, the minimal polynomial of block $\operatorname{diag}\left\{A^{[1]}, A^{[3]}\right\}$ is

$$
\left(\lambda-e^{j \omega T}\right)\left(\lambda-e^{-j \omega T}\right)\left(\lambda-e^{j 3 \omega T}\right)\left(\lambda-e^{-j 3 \omega T}\right) .
$$

Therefore,

$$
G_{1}=\left[\begin{array}{cccc}
\cos \omega T & \sin \omega T & 0 & 0 \\
-\sin \omega T & \cos \omega T & 0 & 0 \\
0 & 0 & \cos 3 \omega T & \sin 3 \omega T \\
0 & 0 & -\sin 3 \omega T & \cos 3 \omega T
\end{array}\right], \quad G_{2}=\left[\begin{array}{l}
0 \\
1 \\
0 \\
1
\end{array}\right]
$$

The compensator together with the plant forms an 8-dimensional system. Again, the feedback gain $\left(K_{1}, K_{2}\right)$ is chosen such that the eigenvalues of the linearized closed-loop system are

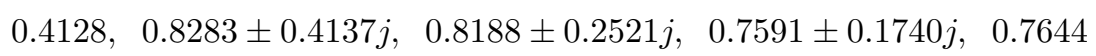

which, again, are obtained by the bilinear transformation from the ITAE prototype design for the continuous-time systems with the cutoff frequency equal to $4.0 \mathrm{rad} / \mathrm{sec}$.

Both the control laws are designed based on the nominal values of the system parameters which are given as follows: $b=12.98 \mathrm{~kg} / \mathrm{sec}, \mathrm{M}=1.378 \mathrm{~kg}, \mathrm{l}=0.325 \mathrm{~m}$, $\mathrm{g}=9.8 \mathrm{~m} / \mathrm{sec}^{2}, \mathrm{~m}=0.051 \mathrm{~kg}$.

First, we compare the performance of three types of controllers, namely, the linear controller, the third order controller and the third order robust controller, for the nominal case, i.e., for the system without parameter perturbations. The frequency of the reference input is fixed at $\omega=0.5 \pi$ while the amplitude $A_{m}$ of the reference input takes $A_{m}=0.75,1.0,1.25,1.5$. Table 1 shows the maximal steady state tracking errors of the closed-loop systems under various control laws for $\omega=0.5 \pi$ and $A_{m}=0.75,1.0,1.25,1.5$. It is seen that the tracking performance of all the controllers are quiet good. The steady state tracking error of the third order robust controller is much smaller than the other two controllers while the third order controller is better than the linear controller. Figure 2 shows the tracking performance of the nominal closed-loop system resulting from the third order controller and the third order robust controller with $A_{m}=1.25$ and $\omega=0.5 \pi$.

Next, we compare the robust performance of the various controllers in the presence of parameter uncertainties with $A_{m}=1.25$ and $\omega=0.5 \pi$. Assume that the parameter 
$b$ is perturbed to $b=12.98+\Delta b$ with $\Delta b=-1.0,-0.5,0.5,1.0,1.5$. Table 2 shows the steady state tracking error of the perturbed closed-loop systems. As shown in Table 2 , the third order robust controller has quiet strong robust performance with respect to the parametric uncertainties. In various cases of the parameter perturbations, the maximal steady state tracking errors are kept small. In contrast, the tracking performance of both the linear and the third order controller greatly deteriorates when the parametric uncertainties are present. Also, while the third order controller performs much better than that of the linear controller in the nominal case, it has no advantage over the linear controller when the parameter uncertainties are present. Figure 3 shows the tracking performance of the perturbed closed-loop system resulting from the third order controller and the third order robust controller with $A_{m}=1.25$ $\omega=0.5 \pi$, and $\Delta b=1.0$.

6. Conclusions. Two types of the robust servomechanism problems, namely, the discrete-time $k^{\text {th }}$-order robust servomechanism problem and the discrete-time robust servomechanism problem, are considered in this paper. While the $k^{t h}$-order robust control renders the closed-loop error output zero up to $k^{\text {th }}$-order of the exogenous signal in the presence of small parameter perturbations, the robust control requires the closed-loop error output tend to zero asymptotically in the presence of small parameter perturbations. The solvability condition of the discrete-time $k^{t h}$-order robust regulation problem is established first based on the Jacobian linearization of the nonlinear plant by using the internal model principle. It is further shown that, if the solution of the discrete regulator equations is a polynomial function of the exogenous signal, then the controller solving the discrete-time $k^{\text {th }}$-order robust servomechanism problem also solves the discrete-time robust servomechanism problem for the same nonlinear system. The example of the inverted pendulum on a cart system demonstrates the effectiveness of the $k^{t h}$-order robust controller in comparison with the $k^{t h}$-order approximate controller when the parameter uncertainties exist in the plant.

\section{REFERENCES}

[1] C. I. Byrnes, F. Delli Priscoli, A. Isidori, And W. Kang, Structurally stable output regulation of nonlinear systems, AutomaticA, 33(1997), PP. 369-385.

[2] J. Carr, Applications of centre manifold theory, LeCture Notes in Control and Information Sciences, Springer-Verlag, New York, 1981.

[3] B. Castillo and S. D. Gennaro, Asymptotic output tracking for SISO nonlinear discrete time systems, Proceedings of the 30th IEEe Conference on Decision and Control, 1991, PP. $1802-1806$.

[4] B. Castillo, S. D. Gannaro, S. Monaco, and D. Normand-Cyrot, Nonlinear regulation for a class of discrete-time systems, Systems \& Control Letters, 20:1(1993), Pp. 57-66.

[5] E. J. DAvison, The robust control of a servomechanism problem for linear time-invariant multivariable system, IEEE Transactions on Automatic Control, 21:1(1976), PP. 2534 . 
[6] C. A. Desoer AND Y. T. WAng, Linear time-invariant robust servomechanism problem: a self-contained exposition, Control And Dynamic Systems, 16(1980), PP. 81-129.

[7] B. A. Francis and W. M. Wonham, The internal model principle of control theory, AutoMATICA, 12(1976), PP. 457-465.

[8] B. A. Francis, The linear multivariable regulator problem, SIAM Journal on Control AND Optimization, 15(1977), PP. 486-505.

[9] R. Gurumoorthy And S. R. SAnders, Controlling nonminimum phase nonlinear system: the inverted pendulum on a cart example, Proceedings of American Control Conference, San Francisco, California, USA, June 1993, PP. 680-685.

[10] J. HuANG, K-fold exosystem and the robust nonlinear servomechanism problem, ASME JouRnal of Dynamic Systems, Measurement, And Control, 120:3(1998), PP. 149-153.

[11] J. HuANG AND C. F. Lin, On the servomechanism problem of discrete-time nonlinear systems, Proceedings of American Control Conference, San Francisco, California, USA, JUNE 1993, PP. 884-848.

[12] J. HUANG AND C. F. Lin, Internal model principle and robust control of nonlinear systems, Proceedings of the 32nd ieEe conference on Decision and Control, December 1993, PP. 1501-1506.

[13] J. HUANG AND C. F. Lin, On a robust nonlinear servomechanism problem, IEEE TrANSACtions on Automatic Control, 39:11(1994), PP. 1510-1513.

[14] J. HuAng AND C. F. Lin, A stability property and its application to discrete-time nonlinear system control, IEEE Transactions on Automatic Control, 39:11(1994), PP. 23072311.

[15] J. Huang And W. J. Rugh, Stabilization on zero-erro manifolds and the nonlinear servomechanism problem, IEEE Transactions on Automatic Control, 37:7(1992), PP. 1009-1013.

[16] J. Huang And W. J. Rugh, An approximation method for the nonlinear servomechanism problem, IEEE Transactions on Automatic Control, 37:9(1992), pP. 1395-1398.

[17] A. IsIdORI, A remark on the problem of semiglobal nonlinear output regulation, IEEE TRANS. on Automatic Control, 42(1997), pP. 1734-1738.

[18] A. Isidori And C. I. Byrnes, Output regulation of nonlinear systems, IEEE TransaCtions on Automatic Control, 35:2(1990), PP. 131-140.

[19] H. KhaLIL, Robust servomechanism output feedback controllers for feedback linearizable systems, Automatica, 30(1994), PP. 1587-1589.

[20] H. KhaLIL, On the robust servomechanisms for minimum phase nonlinear systems, INT. J. Robust Nonlinear Control, 10(2000), PP. 339-361.

[21] D. Wang And J. Huang, Solving the discrete-time output regulation problem with Taylor series Method, Proceedings of the Chinese Control Conference, Hong Kong, December 2000, PP. 700-704. 
TABLE 1

The maximal steady state tracking errors of the nominal system.

\begin{tabular}{|c|c|c|c|c|}
\hline \hline Amplitude & $\omega$ & Linear & Third Order & Third Order Robust \\
\hline 0.75 & $0.5 \pi$ & 0.0095 & 0.0002 & 0.0000 \\
\hline 1.00 & $0.5 \pi$ & 0.0226 & 0.0007 & 0.0000 \\
\hline 1.25 & $0.5 \pi$ & 0.0446 & 0.0021 & 0.0002 \\
\hline 1.50 & $0.5 \pi$ & 0.0788 & 0.0053 & 0.0008 \\
\hline
\end{tabular}

TABLE 2

The maximal steady state tracking errors of the perturbed system with $A_{m}=1.25$ and $\omega=0.5 \pi$.

\begin{tabular}{|c|c|c|c|}
\hline \hline$\Delta b$ & Linear & Third Order & Third Order Robust \\
\hline 0.00 & 0.0446 & 0.0021 & 0.0002 \\
\hline-1.00 & Unstable & Unstable & 0.0014 \\
\hline-0.50 & 0.1502 & 0.1408 & 0.0006 \\
\hline 0.50 & 0.1787 & 0.1792 & 0.0001 \\
\hline 1.00 & 0.4125 & 0.4150 & 0.0001 \\
\hline 1.50 & 0.7367 & 0.7399 & 0.0009 \\
\hline
\end{tabular}

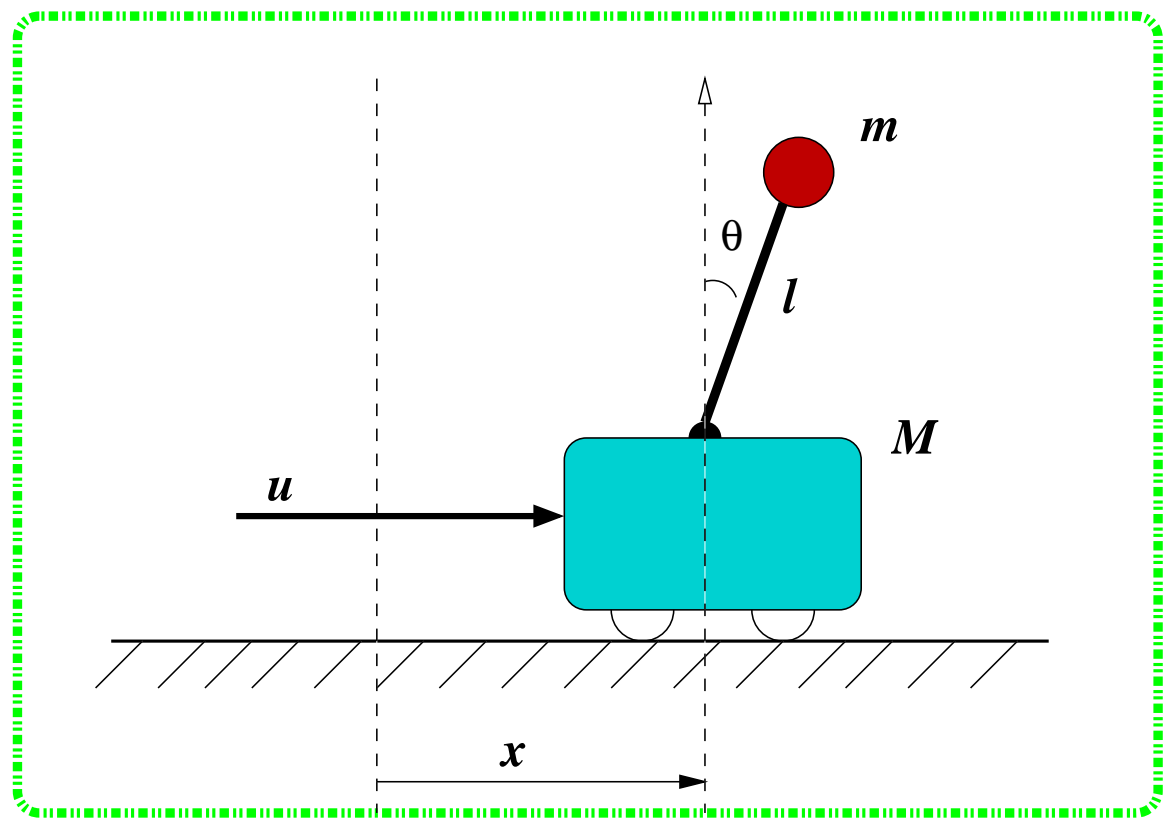

FIG. 1. Inverted Pendulum on a cart system 


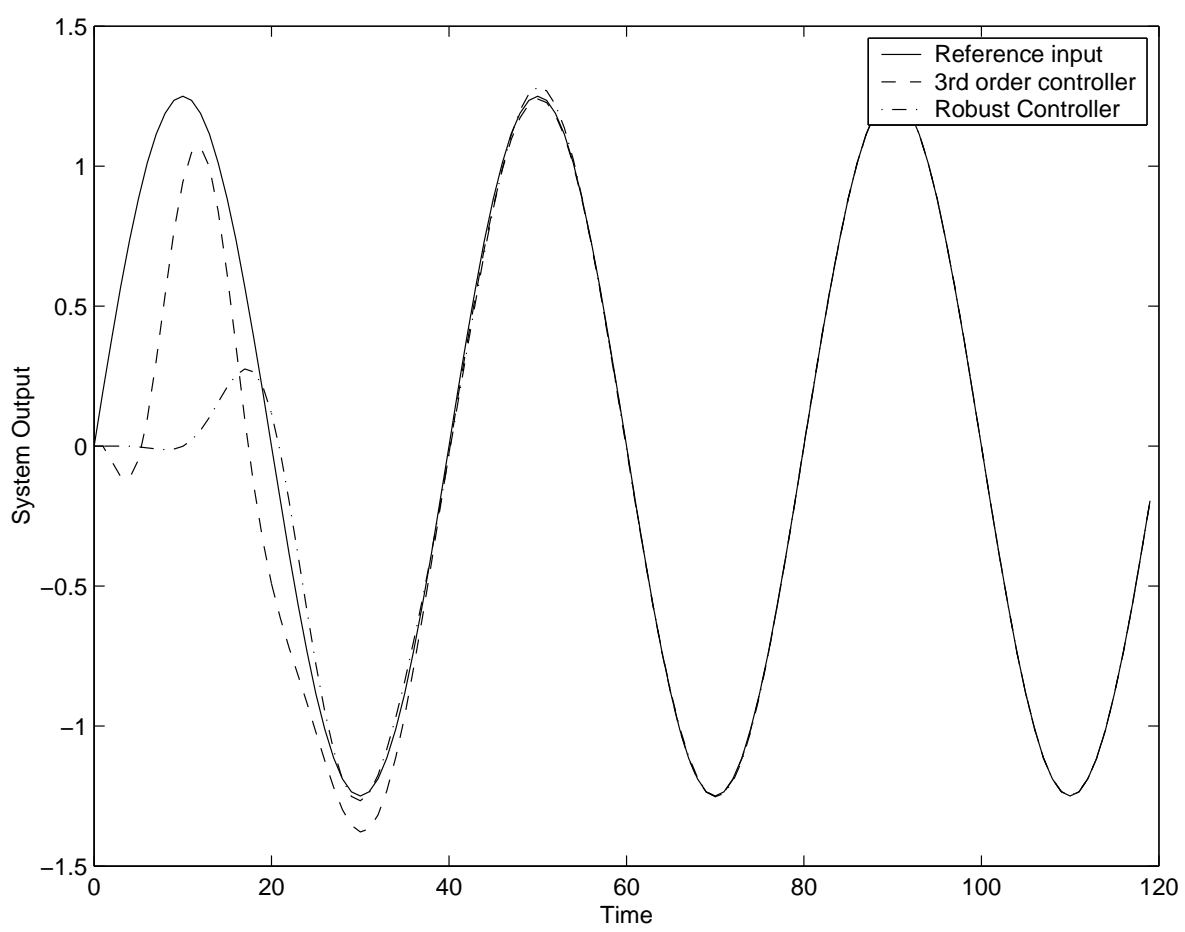

FIG. 2. Tracking performance: nominal system with $A_{m}=1.25$ and $\omega=0.5 \pi$

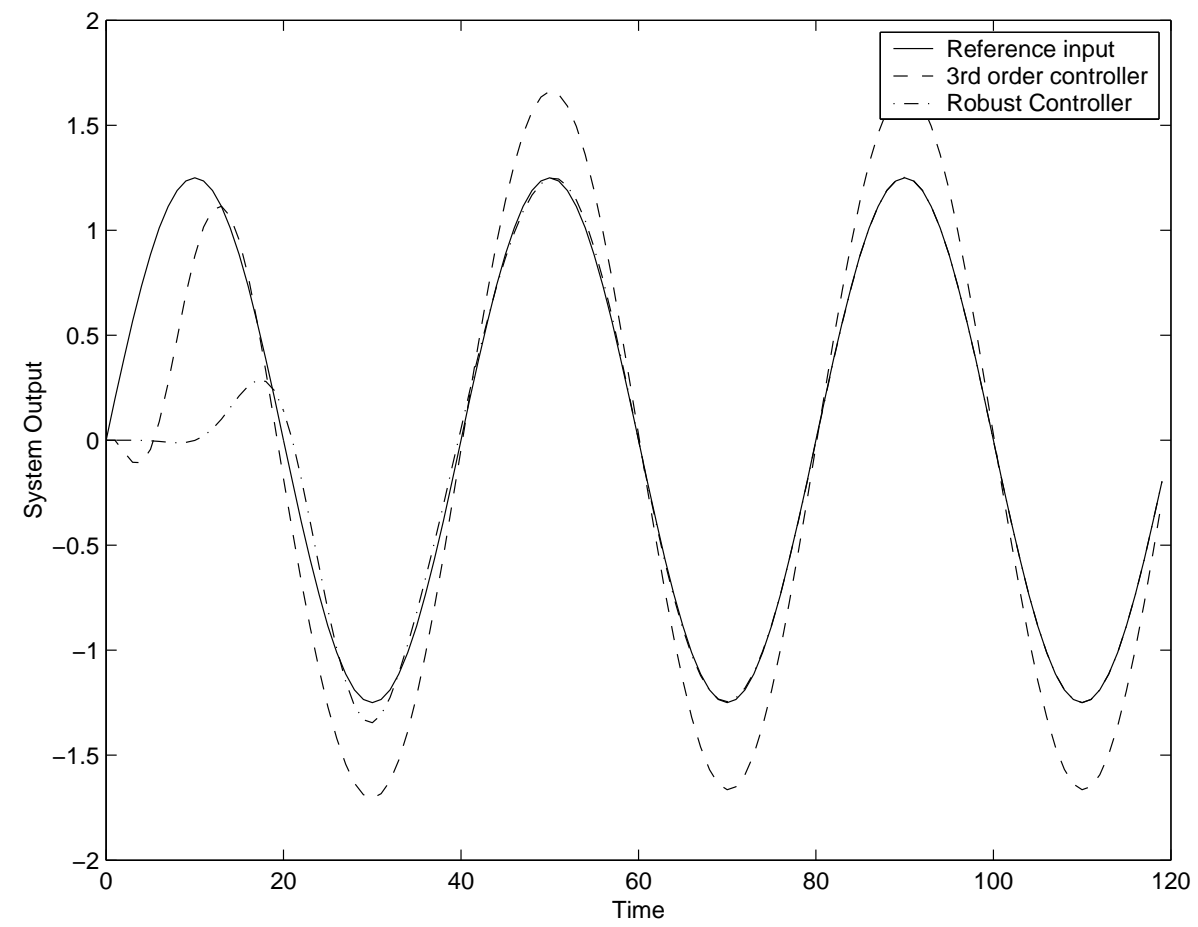

FIG. 3. Tracking performance: perturbed system with $A_{m}=1.25, \omega=0.5 \pi$ and $\Delta b=1.0$ 
